\title{
Electrical Injury: Histopathologic Findings on One Case
}

by

\author{
M. Kitano, S. Tokufuji \\ Department of Pathology, Kyushu Rosai Hospital \\ Kitakyushu-shi, Japan 800-02 \\ M. Kawashima, M. Maniwa \\ Orthopedic Surge1 y, Kyushu Rosai Hospital \\ and \\ T. Nagao \\ Rehabilitation Medicine, Kyushu Rosai Hospittal
}

\begin{abstract}
Histopathologic findings on electrical injury of excised specimens from a six-year-old boy were described. Deep tissue necrosis was extremely marked and it was associated with vascular alteration.

Coagulation necrosis of the vessels walls together with thrombogenesis was one of the most important changes in the present case.
\end{abstract}

The principal pathologic change caused by electricity is coagulation necrosis of the tissue as a result of sufficient electrical current flow. Tissue damage is produced by heat, and the heat production is related to current flow (Joule effect) ${ }^{4}$. In contrast to the usual form of thermal burn, the electrical injury is frequently deep and accompanied by impairment of nerve, blood vessels, muscles and others. The resistance of various tissue to flow of current increases in the following order: nerve, blood, muscle, skin, tendon, fat and bone ${ }^{2)}$. In the blood vessels, which offer little resitance to flow of electric current, the necrosis occurs easily and extensively.

The vascular pathology in the cases of electrical injury explains why deep tissue necrosis can occur at a considerable distance from the contact point, entrance or exit of the current.

The present study deals chiefly with histopathologic changes on excised specimens from a case of electrical injury. And the final purpose of the study is to make clear the role of the vascular lesions in electrical injury.

\section{Case Report}

A six-year-old boy was exposed to a potential 6,600 volts. Immediately after, he fell dow in a shock state and was treated by emergency doctors. Three days after the injury, he was transferred to Kyushu Rosai Hospital. On admission, slight anemia and slight jaundice were pointed out. Whole parts of the left hand was mummified, and the left forearm 
lacked sensation and was pulseless. The right middle finger was necrotic with skin discoloration till the PIP joint and multiple bulla-formation was observed in the entire skin of the right hand. Moreover, the bulla-formation in slight degree was found in the bilateral fossae cubitii et axillae.

While the general condition became better, the necrotic lesion of the left hand extended to the antebrachial region and the discolored area of the skin was clearly demarcated at the site of $1 / 3$ distal of the forearm (Fig. 1).

Amputation of the left forearm at the site of $1 / 2$ distal and the right middle finger at the site of PIP joint were done, 15 days and 22 days after the injury, res-

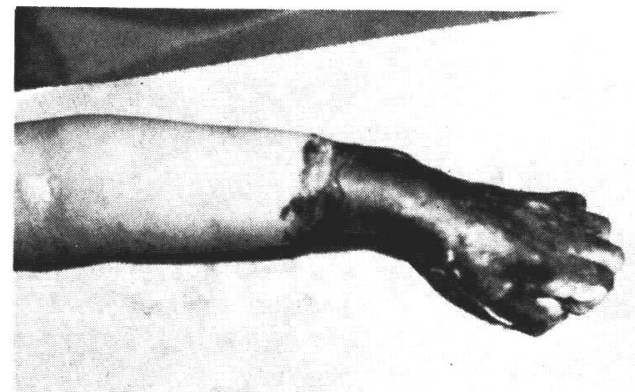

Fig. 1: Mummified left forearm at the time of surgical operation. Discoloration of the overlying skin is marked. pectively.

\section{Histopathologic Findings}

Excised specimens were fixed in $10 \%$ formalin. Prepared paraffin-sections were stained with H-E, PAS, Elastica Van Gieson, Azan Mallory, PTAH, Prussian blue reaction for ion and Rubeanic acid reaction for copper.

The principal histologic change was wide spread coagulation necrosis of the epidermis, corium, subcutaneous adipose tissue, skeletal muscles, nerve fibers, etc., of the examined sections (Fig. 2).

Almost all walls of the arteries, arterioles, veins and venules in both superficial and deep layers seemed necrotic and the loss of stainability of cellular nuclei of the vessel walls was marked. Construction of the vessels, however, was relatively-well preserved. Large amounts of erythrocytic thrombi filled the lumina of the affected vessels resulting in marked dilatation of the vessels. Expansion of the arterial wall was occasionally extensive (Fig. 3).

The muscular layer showed patchy hemorrhage and small nutrient vessels were extremely occluded by thrombi (Fig. 4).

In the bone marrow cavity, dilated sinusoids including erythrocytic thrombi were observed and the bone marrow tissue was necrotic. Almost all osteocytes in bony lacunae were unstainable showing cellular death. Massive but focal periosteal hemorrhage was observed in the left ulnar bone (Fig. 2).

In the border zone between the necrotic area and the intact part of the left forearm, proliferation of granulation tissue including numbers of siderophages was rather abundant. Leukocytic infiltration was also seen but in slight degree. Organization of the thrombi and recanalization of the thrombotic vessels were prominent here (Fig. 5).

Searching for copper ion was unsuccessful in any of the specimens.

\section{Discussion}

The principal pathologic change caused by electricity is coagulation necrosis of the tissue.

$$
-39-
$$




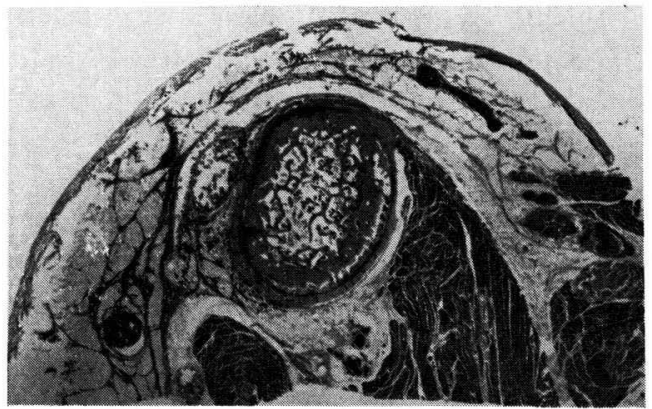

Fig. 2: Coagulation necrosis of superficial and deep tissue of the left forearm (ulnar side). Thrombosis of the cutaneous veins is dominant. Hemorrhage is marked in the periosteum of the ulnar bone.

$\mathrm{H}-\mathrm{E}, \times 13$.

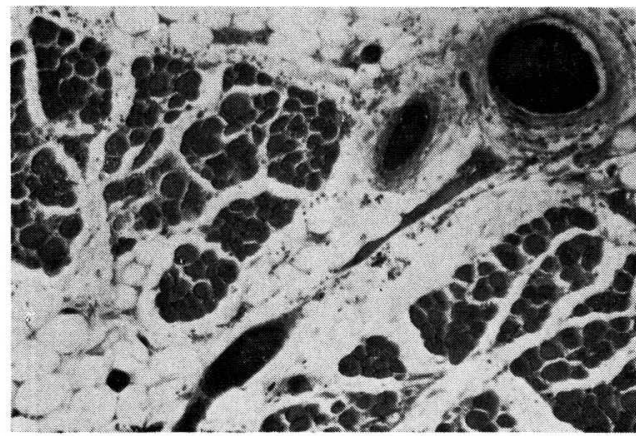

Fig. 4: Coagulation necrosis of the skeletal muscle (left hand). Muscle fibers swell and nuclei of them show complete loss of stainability. Thrombosis is dominant in the nutrient vessels. Hemorrhage is also noted. H-E, $\times 90$.

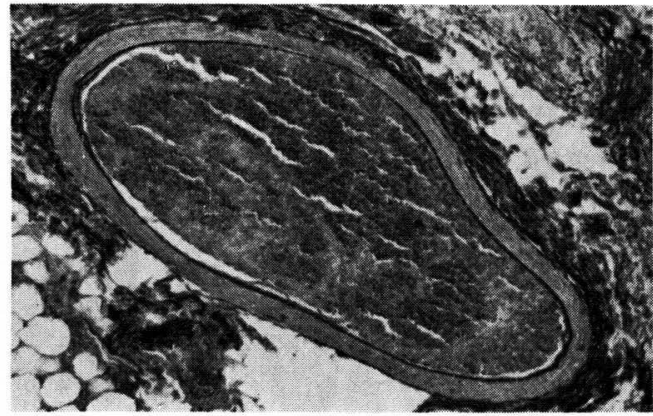

Fig. 3: Marked dilation of a branch of the ulnar artery (left forearm). Coagulation necrosis of the arterial wall is associated with stretching and disintegration of the elastic laminae. Thrombus fills the lumen. Elastica Van Gieson, $\times 90$.

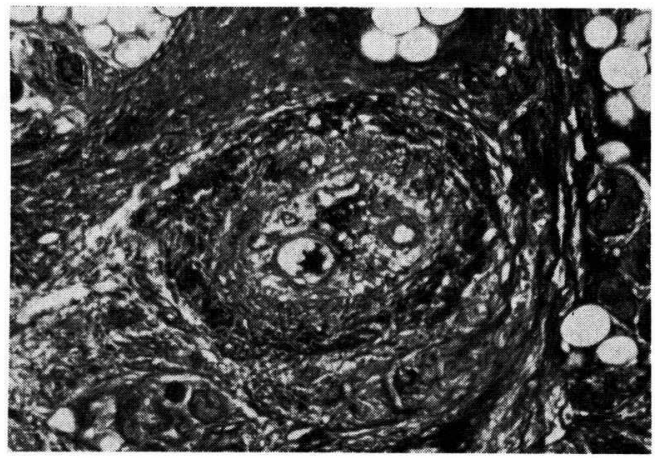

Fig. 5: Recanalization of thrombosed small artery in the border zone between the damaged area and the intact part of the left forearm.

Azan Mallory, $\times 90$.

In contrast to the thermal burn, the electrical injury is usually deep and results in muscle damage. Pathologic examination of the present case reveals extensive coagulation necrosis of the muscle, microscopically characterized by interstitial hemorrhage, swollen muscle fibers of which nuclei show complete loss of stainability, and erythrocytic thrombi occluding many muscular vessels.

In the blood vessels, which offer little resistance to flow of electric current, the necrosis occurs in the vessels walls ${ }^{4)}$. Vascular lsions dominate the clinical picture in many electrical injuries ${ }^{16) 7)}$. Such injuries often consist or a limited cutaneous burn associated with deep muscle damage of variable extent. Many investigators assess the role of vascular injury in the pathogenesis of electrical injury. They recorded that the arterial changes in the vicinity of electrical burns are due to heat. The vessels become dilated and spontaneous rupture may occur. As it was already pointed out, thrombi are apt to form within the 
damaged vessels resulting in ischemic necrosis of tissue ${ }^{5)}$.

The effect of vascular damage is also well demonstrated by the clinical course of the present case. The fairly exensive progression was seen in the left burned forearm day by day.

Major vascular alterations relating to muscle injury appear to be confined to the smaller nutrient muscular arteries and arteriolar branches. The muscle necrosis associated with electrical injuries is produced when the electrical energy is converted to thermal energy (4)6). The extremely high temperatures produce coagulation necrosis and occlusion of small vessels, but commonly blood flow in large arteries in a current pathway is sufficient to dissipate heat and prevent clotting. Small nutrient muscular branches are extremely susceptible to thrombogenesis at the time of exposure to high temperatures, and the pathologic examinations of the electrically injured muscle confirm the presence of small arterial and microarterial thromboses ${ }^{3}$.

Bone is a poor electrical conductor, but, according to experimental work of Hunt ${ }^{3)}$, when bone and muscular tissue are supplied with the same temperatures during application of sufficient electrical energy, bone retains heat longer than muscle. The slower dissipation of heat may be possibly responsible for the periosseous tissue necrosis. In the present case, massive but focal hemorrhage was found in the periosteum of the left ulnar bone and this fact may point out that the periosseous vessels were damaged more than superficially situated vessels.

\section{Acknowledgment}

The authors are grateful to Emeritus Prof. Dr. Amako, director of Kyushu Rosai Hospital, Dr. Iwabuchi, chief of the Dept. Orthop. Surg. of Kyushu Rosai Hospital and Prof. Boles, staff of Kyushu Rehabilitation college for their excellent guidance. Thanks are due to $\mathrm{Mr}$. Hatta and Mrs Gonno, members of Kyushu Rosai Hospital for their great cooperation.

\section{References}

1) Artz, C. P.: Changing concepts of electrical injury. Am. J. Surg. 128:600-602, 1974.

2) DiVincenti, F. C. et al.: Electrical injuries: A reviewof 65 cases. J. Trauma 9: 497-505, 1969.

3) Hunt. J. L. et al.: Vascular lesions in acute electric injuries. J. Trauma 14: 461-470, 1974.

4) Jaffe, R. H. et al.: The effect of electric currents on the arteries: A histologic study. Arch. Path. 7: 244-252, 1929.

5) Marchesi, V. T.: Disturbance of body water and circulation of blood. Pathology (edited by Anderson, W. A. D. \& Kissane, J. M.) 7th Ed. pp 148-192, Mosby, St. Luis, 1977.

6) Shimada, N.: Dengekisho (Electrical injury) in Nippon-Geka-Zensho 2, Kanehara-Nankodo, Tokyo, 1954 (in Japanese).

7) Skoog, T.: Electrical injuries. J. Trauma 10:816-830, 1970. 